\title{
GRILLES TOURNANTES ET GRILLES ÉLECTRIQUES AUX ÉTATS-UNIS EN 1931
}

\author{
Par M. GALLOIS \\ Conservateur des Eaux et Forêts en retraite \\ et M. DE DROUIN DE BOUVILLE \\ Inspecteur principal des Eaux et Forêts en retraite.
}

On sait l'importance des mouvements de migration des Salmonides potamotoques dans les cours d'eau du Far-West, tributaires du Pacifique, dont le plus important est la Columbia, avec son vaste bassin s'étendant sur les quatre Etats d'Idaho, Montana, Oregon ct Washington. Ces fleuves côtiers et leurs aflluents sont fréquentés surtout par l'Oncorhynque chinook (Oncorhynchus tschawitscha Walbaum) et le Saumon tête d'acier (Salmo Gairdneri Richardson) qui les descendent par myriades, à l'état de tacons, pour les remonter, en masses imposantes, après la période de croissance en mer qui précède la maturité sexuelle.

Lors de l'avalaison, ces poissons courent le risque de s'engager dans les artères maîtresses des réseaux d'irrigation, risque grave ; lors du voyage nuptial, ils ont tendance à pénétrer dans les canaux de fuite des usines hydrauliques où les attire le courant vif des turbines, et, dès lors, ont grandes chances de ne pouvoir parvenir aux régions montagneuses où se trouvent les frayères. Il importe donc d'interdire à ces animaux voyageurs, soit à l'aller, soit au retour, l'accès des dérivations où ils se fourvoieraient volontiers. Pour cela, on a recours à deux moyens : la grille tournante et la grille électrique.

Des modèles satisfaisants de ces deux sortes d'appareil ayant été réalisés en 1929, nous en avons donné, voici deux ans, la description complète ( 1 ). Il a été rendu compte, ensuite, des progrès réalisés en ig3o (2).

Le moment est venu, aujourd'hui, de faire connaître, d'après la plus récente publication du Bureau of Fisheries de Washington (3), - dont le rédacteur est M. Elmer Higgiss, chef de la Division des Investigations scientifiques -, comment a évolué, en $193 \mathrm{I}$, la question de la protection des migrateurs amontants ou dévalants contre les dangers résultanit de l'amé. nagement hydraulique des cours d'eau à fins agricoles ou industrielles.

(1) Nouveaux dispositifs pour interdire aux poissons l'accès des dérivations hydrauliques. - Bulletin : - $n^{\circ} 39$, septembre $19^{3} 1$, p. $73 ;-n^{0}$ il, Novembre $\mathbf{1 9}^{3} \mathrm{I}$, p. $133 ;-n^{\circ} 42$, Décembre Ig3 I, p. 169 .

(a) Les grilles électriques aux Etats-Unis. - Bulletin $n^{\circ} 48$, Juin 1932, p. 364 .

(3) Progress in biological inquiries, appendix III to report of the Commissionner of Fisheries for the fiscal year 1932. - Government printing Office, Washington, rg32. 
Rappelons que les spécialistes chargés des études et contrôles sont toujours : M. Shirley Baker, Ingénieur Conseil à San-Francisco, et M. U. B. Gir roy, Ingénieur civil.

Nous traiterons, distinctement et successivement, des grilles tournantes, qui donnent pleine satisfaction, et des grilles électriques, objet actuel d'une défaveur plutôt imméritée.

\section{I. - Grilles tounnantes}

C'est l'Oregon Game Commission qui, en $19^{3} \mathbf{I}$, pour interdire aux poissons l'entrée des réseaux d'irrigation sans arrêter feuilles, herbes et détritus charriés, préconisa l'installation, à la prise d'eau, de cylindres en treillis métallique virant autour d'un axe horizontal.

Depuis 1930, la revolving screen est au point, le type étant celui adopté pour le canal Ahtanum (I). Aussi, dès l'année suivante des appareils similaires étaient en cours ou en instance de pose sur les dérivations suivantes, classées par ordre d'importance croissante :

Arnold Canal (Oregon) .... débit maximum : 2.800 litres-seconde.

Farmers Irrigation Ditch-

Hood river (Oregon).... - $\quad-\quad 2.800$

Swalley Canal (Oregon).... - $\quad-\quad$ - $\quad 3.500$

Tumalo Canal (Oregon) ... - - $\quad-\quad 4.200 \quad-$

Jocko Canal (Montana) ... $\quad$ - $\quad-\quad 8.400 \quad-$

Pilot Butte Canal (Oregon).. - $\quad$ - $\quad$ I1.600 -

C. O. I. Canal (Oregon).... - $\quad$ - $\quad$ I8.200 -

Sun River Slope Canal
(Montana) $\ldots \ldots \ldots \ldots \ldots \ldots-40.200-$

On voit, par ce tableau, que le domaine d'application des grilles tournantes s'est rapidement et largement étendu, car, un an plus tôt, on n'envisageait guère la possibilité de leur emploi sur des canaux de section supérieure à celui d'Ahtanum, qui a, en moyenne, $4 \mathrm{M}$. de largeur et I M., 20 de profondeur, avec un débit ne dépassant pas 2.225 litresseconde.

Comme on le verra par la suite, on se trouve incité à recourir de préférence, aujourd'hui, aux dispositifs mécaniques, l'opinion publique ayant des préventions, plus instinctives que raisonnées, contre les barrages électriques.

M. Higgins est fondé à qualifier de très hardi (most ambitious) le projet de la Sun river qui, à raison de son importance, mérite brève mention.

L'eau de la rivière en question, retenue par un barrage qui coupe une gorge étroite, est dirigée sur le réservoir de Pishkun, d'où se détache l'artère principale du périmètre à irriguer : le Sun River Slope Canal, dont la section normale a 9 M., I4 de largeur sur fond et 3 M., 5o de profondeur.

(1) La description détaillée, avec plan, coupes et photographie a êté donnée dans le Bulletin ; - voir $n^{\circ} 39$, Septenbre $193 \mathrm{r}$, p. 77 . 
La grille tournante est installée à $800 \mathrm{M}$. environ de l'origine, à raison de l'existence d'une dépression naturelle du sol sur laquelle le courant s'étale, perdant de sa vitesse, ce qui est une ciroonstance favorable. Sur cet emplacement, cinq tambours sont alignés, ayant, chacun, १ M., 27 de largeur et 3 M., 96 de diamètre ; leur enveloppe est un filet métallique maillé à 16 millimètres. Le mouvement de rotation est obtenu au moyen de trois roues à palettes montées immédiatement au-dessous des tambours, la liaison étant assurée par des chaînes à maillons engrenant sur pignons dentés.

Pour donner idée de l'importance de l'ouvrage, terminons en indiquant que le massif de béton servant d'assise a un volume de Io I/ 2 mètres cubes ; son armature pèse approximativement 22.700 kilogrammes. Enfin, les grilles et roues ont exigé, pour leur construction, 2 I.3oo kilogrammes d'acier.

\section{II. - Grilles electriques}

Pour le Bureau of Fisheries, le type définitif de l'electric fish screen s'est trouvé fixé, lui aussi, à la fin de $\mathrm{x} 930$, à la suite d'expériences dont les principales furent celles de l'Old Indian Canal (Washington) et de la pisciculture de Fort Klamath (Oregon) (i). Le modèle officiel est constitué par deux câbles parallèles supportant des élcctrodes immergées. Les meilleurs résultats ont été enregistrés avec des tuyaux de fer galvanisé, convenablement lestés, équidistants de I M., 22, mesure prise de centre à centre, l'écartement des câbles étant de r M., 83. On applique à ce dispositif un courant alternatif à 60 périodes, d'une tension de 55-65 volts, réglée par un transformateur spécial, dont l'inventeur est le Professeur Mac Millan, titulaire de la chaire de technique électrique à l'Oregon Stat, College (2).

Mais les événements dont il va être rendu compte sommaire prouvent qu'il ne suffit pas, pour réussir, d'avoir un appareil bien conçu et étudié, car, au pays même où fut imaginée la grille électrique, elle ne s'est pas imposée, loin de là.

Les principaux travaux tendant à sa mise au point ont été effectués à l'usine hydraulique de Gold Ray, sur la Rogue river, tributaire du Pacifique (3). C'est la Centrale de la California-Oregon Power Company, peu distante de Medford (Oregon).

On s'était attaqué, là, à un double problème, car on se proposait de condamner la dérivation tant à I'entréc qu'à la sortie, autrement dit d'arrêter les migrateurs aussi bien à la descente qu'à la remonte.

Une première grille fut donc établie à l'origine du canal des turbines. Mise en charge le 3 Juin 1929 , elle donna des mécomptes qui contraignirent à modifier du tout au tout son ogencement. Mais la nouvelle ins.

(1) Voir Bulletin : - $\mathrm{n}^{\circ} 48$, Juin 1932 , p. 367 .

(2) Voir Bulletin : $-n^{\circ} 42$, Décembre 1931, p. 176

(3) Voir, pour ce qui concerne les essais de $19^{2} \&-1930:-$ Bulletin, $-\mathrm{n}^{\circ} 42$, Décembre $19^{31}$, p. $17^{3} ;-\mathrm{n}^{\circ} 48$, Juin $19^{32}$, p. 366 . 
tallation, mise à l'épreuve en r 930 , se révéla peu efficace. M. HıgGrvs opine qu'elle était trop peu distante d'une herse en acier retenant les corps flottants. Mais on sait que l'insuffisance des dispositifs électriques, quand il s'agit d'arrêter les poissons se laissant aller au fil de l'eau, a été signalée par le docteur HoLzer (I).

Quoi qu'il en soit, quand on vit, à Gold Ray, de nombreux tacons franchir la double ligne d'électrodes, on imagina d'éclairer très vivement l'entrée de la passe destinée à faciliter à ces poissons le franchissement du barrage, pour les attirer, par fascination. Ce fut un succès ; la passe devint très attractive et, du coup, l'electric screen devint inutile.

Le second des dispositifs, à savoir celui barrant le canal de fuite aux migrateurs amontants, a occasionné pire déception aux spécialistes du Bureau of Fisheries.

Remémorons qu'en ${ }_{1928}$ appel avait été fait à ces techniciens par la California-Oregon Power Company et que, sur leur suggestion, on avait installé, l'année suivante, au travers d'une section mouillée de 22 M., 85 sur I M., 68, un appareil type Burkex comprenant un tuyau de fer galvanisé de 6 I/4 centimètres de diamètre et une frange de chaînettes électrodes, à l'écartement de 45 centimètres, suspendues à un câble tendu audessus du niveau liquide. Mais, lors de la remonte de I93o, beaucoup d'Oncorhynques chinook et de Saumons tête d'acier franchirent l'obstacle et certains furent foudroyés à l'aplomb du gruond element (2).

On se hâta de substituer à cet appareillage défectueux celui du Professeur Mac Mullan à deux franges d'électrode. Il entra en fonctionnement à l'automne et se révéla efficace.

Au printemps de r93 I, tout alla bien jusqu'au 25 Mai, date à laquelle intervint une réclamation de la Jackson County Game Protective Associalion, groupement de pêcheurs sportifs ayant son siège à Medford. Elle dénonçait l'électrocution à Gold Ray de migrateurs par centaines.

Une enquête, à laquelle il fut procédé dès le surlendemain, confirma la réalité des pertes ; il fut constaté, en outre, que des poissons se trouvaient dans la section interdite du canal de fuite, en amont de la grille électrique.

Ces incidents ayant été relatés par la presse, les aménagements de Gold Ray furent âprement critiqués.

Il parut prudent de réduire immédiatement de 65 à 50 volts la tension du courant appliqué et des observations furent décidées. Mais elles ne purent être entreprises, car, le $3 o$ Mai, les dirigeants de la Jackson County... Association, exerçant, à l'insu des représentants locaux du Bureau of Fisheries, une pression énergique sur le Vice-Président et Direc. teur général de la California-Oregon Power Company, obtinrent qu'on arrêtât le fonctionnement de l'installation suspecte. Le ${ }^{\text {er }}$ Juin, les électrodes furent enlevées.

Le lendemain et le surlendemain, les experts de l'Administration confé-

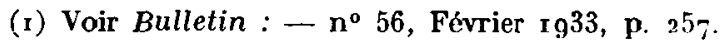

(2) Voir Bulletin : $-n^{\circ} 42$, Décembre 1931 , p. $7_{7}^{5} ;-n^{\circ} 48$, Juin $19^{3}$, p. 367 . 
rèrent avec les délégués du groupement sportif et de la société exploitante. A ce moment, I.200 à r .50o migrateurs stationnaient dans la dérivation usinière, où ils avaient pénétré depuis la suppression de la grille électrique. Il y avait là une preuve d'efficacité ; néanmoins, les mandataires des pêcheurs à la ligne persistèrent à s'opposer à son rétablissement.

Au cours des entretiens, on apprit que, le i6 Avril et le 29 Mai, sur la ligne de 66.ooo volts entre la Centrale de Gold Ray et la Sous-Station de Grants-Pass, s'étaient produits des courts-circuits par l'intermédiaire de la Rogue river. Ceci expliquait l'électrocution d'un grand nombre d'Oncorhynques dans cette section du cours d'eau, où, à l'époque, se manifestait un fort courant de remonte.

Peu après, l'Oregon Fish Commission, informée que des troubles dans la distribution d'énergie électrique étaient survenus à diverses reprises dans le passé, même avant l'installation de la grille, s'enquit auprès de la California-Oregon Power Company. Elle obtint communication des relevés relatifs aux courts-circuits pour une période de cinq années et les transmit au Bureau of Fisheries qui les communiqua, pour examen et avis, au Professeur MaC Millax.

Ce dernier se rendit sur les lieux pour une expertise minutieuse au cours de laquelle il s'attacha spécialement à vérifier l'isolement des conducteurs et du transformateur, qui fut trouvé parfait. Deux rapports en date des 22 Aout et 4 Octobre $19^{3}$ I mirent, en conséquence, la grille électrique hors de cause. Les accidents survenus en Avril-Mai étaient imputés à des courants à terre, dérivations accidentelles de la ligne à 60.000 volts, occasionnées par des réparations exécutées sans précautions suffisantes. Les franges d'électrode n'avaient pu recevoir, ni transmettre au cours d'eau ce courant à haute tension et il convenait de reconnaître qu'elles avatent rempli leur rôle protecteur et sauvé bon nombre de poissons.

De ces conclusions, M. Higgins s'autorise pour déplorer leur suppression. Mais l'Administration, dont il est l'avocat, n'est rnalheureusement pas parvenue à faire partager aux pècheurs à la ligne sa conviction que la grille de Gold Ray était, non seulement inoffensive, mais utile.

Aux dernières nouvelles rien ne subsistait donc, à la Centrale de Gold Ray, des installations perfectionnées tendant à aiguiller dans la bonne direction, soit à la descente, soit à la montée, les Salmonides migrateurs de la Rogue river. Après tous les essais faits en collaboration par le Bureau of Fisheries et la California-Oregon Power Company, après tant de dépenses engagées, il n'y a pas à se dissimuler qu'on enregistrait un échec, qui, pour n'être que moral, - la technique étant sauve, - ne facilitera pas une extension de l'emploi des grilles électriques.

Il y a, au surplus, un autre obstacle qu'une opinion publique butée, à savoir les brevets pris par les inventeurs, d'où majoration prohibitive du cout des installations.

Bref, au moment oủ M. Hrgiss a établi son compte rendu, il ne restait plus en fonctionnement que les premiers appareils mis en scrvice, ceux 
équipant divers réseaux d'irrigation du bassin de la Yakima river, affluent de la Columbia (W ashington).

Ces installations, précédemment décrites (I), n'ont pas subi de modifications dignes de mention, sauf celle du Sunnyside Canal qui a dû être déplacée el modiliée en s $9^{3}$ I comme l'avait êté, l'année précédente, celle du Wapato Canal. Elle a été transportée, vers l'amont, sur un emplacement où le courant est moins rapide ; et on a substitué, au système ì ground element, celui comportant deux franges d'électrodes. Enfin on a établi, perpendiculairement à la rive de la Yakima, une grille auxiliaire de $7 \mathrm{M}$., 32 de longueur. Son rôle est de donner un avertissement préliminaire aux tacons qui arrivent au voisinage de la prise d'eau. On espère ainsi les en détourner avant qu'ils soient happés par un courant susceptible de les entrainer.

On contrôle l'efficacité des barrages électriques, dans le bassin de la Yakima, par des dénombrements des poissons trouvés dans les périmètres d'irrigation quand l'abreuvage a pris terme.

En I931, comme les années précédentes, ces vérifications ont eu lieu, sous la surveillance attentive de l'Inspecteur local du Bureau of Fisheries.

C'est ainsi que, dans la zone du Sunnyside Canal, qui a été arrosée du 20 Février au 20 Octobre, le débit à plein étant de 4.200 litres-seconde, on a trouvé $7^{5}$ Saumons, 807 Truites et 36.300 poissons blancs. Comme pré. cédemment, parmi ces derniers, certains types prédominent : - Carpiodes Rafinesque, Ptychocheilus Agassiz, Acrocheilus Agassiz... Leur abondance prouve qu'ils ne sont pas arrêtés par les lignes d'électrodes et il a été observé qu'ils les franchissent à l'état de fretin, ayant seulement 35-4o millimètres de longueur. On sait, depuis longtemps, que de tout petits poissons ne sont pas incommodés par des courants qui se manifestent mortels pour eux quand ils deviennent grands. L'explication de ce phénomène, au premier abord paradoxal, a été donnée dernièrement par le Docteur Holzer ; elle se déduit de la constance, pour une espèce donnée, de la tension de conformation provoquant la narcose ( $\mathrm{I}$ ).

Par ailleurs, du 18 au 2 I Juin, à une époque où de grandes bandes de tacons dévalaient au barrage de Sunnyside, on établit un grillage au travers d'une des rigoles de la partie inférieure du réseau. Il retint seule. ment 36 Saumoneaux, et aucune Truite, contre $\varepsilon_{1}$ o poissons divers.

Une dêmonstration plus convaincante encore de l'efficacité de la grille électrique résulte d'une comparaison faite entre le périmètre du Tieton. Canal, qui en comporte une, et celui du Selah Naches Canal, qui en est dépourvu. Les conditions sont similaires de part et d'autre.

Dans le second de ces pérìmètres, it fut recueilli, en deux opérations : -4.573 Saumons et -665 Truites ; soit au total : 5.238 Salmonides.

Or, dans le réseau de Tieton, on n'en trouva que $5 \times 4$ et encore le courant n'avait-jl été appliqué au dispositif de protection que durant I 4 I jours, alors que la saison d'irrigation avait duré 250 jours.

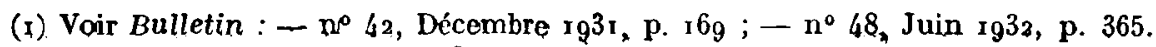

(z) Voir Bulletin : - no 56, Fêvrier 1933 , p. 256 . 
Il est permis de déduire de là que l'electric screen vaut mieux que son actuelle réputation dans l'Oregon. Toutefois, elle n'est pas, elle ne peut être, comme la grille tournante, efficace à cent pour cent. Elle n'arrête guère la blanchaille, ce qui est sans grande importance, mais retient la grande majorité des tacons et rend, par là, l'essentiel du service qu'on lui demande.

Dégageons, en terminant, les leçons des expériences américaines.

La grille tournante est un instrument simple, robuste, commode, relativement peu conteux ; il remplit au mieux son office et n'exige qu'un minimum de surveillance et d'entretien. Inscrivons encore à son crédit la possibilité de l'utiliser sur un emplacement quelconque, même si on n'est pas à même d'y aménager une chute, du moment où on peut y amener le courant électrique pour actionner une dynamo motrice.

La grille électrique, en dépit d'incontestables progrès réalisés depuis son invention, et bien que les règles de sa construction aient été déterminées en r931 par le Docteur Holzer, est un appareil plutôt délicat sur lequel il est prudent d'exercer un contrôle vigilant. Le Bureau of Fisheries, vu l'hostilité des pêcheurs à la ligne, a renoncé à leur emploı après avoir fondé sur lui de grands espoirs (I). Il les délaisse, d'ailleurs à contre-cœur ; aussi sied-il ne ne pas condamner une méthode ingénieuse de sauvegarde des poissons migrateurs parce qu'à Gold Ray son application a soulevé des protestations insuffisamment justifiées. Il ne faut pas oublier, que, sur les dérivations industrielles, les barrages d'électrodes ont le capital avantage de ne pas provoquer de remous appréciable et, par conséquent, d'éviter des pertes de charge.

On se trouvera donc bien, dans certains cas, de recourir à ces dispositifs, surtout s'il s'agit d'intercepter à son terminus un canal de fuike, mais ils devront être établis avec grand soin et, surtout, un souci scrupuleux du parfait isolement, qui sera à vérifier fréquemment. Toutes précautions prises, il ne faudra pas s'émouvoir si quelques Saumons ou Truites franchissent l'obstacle, au risque de leur vie. Il ne faut pas demander à l'electric screen plus qu'elle ne peut donner.

Une dernière conclusion est que le voisinage immédiat d'une ligne à haute tension constitue un danger pour les rivières. En cas de ruptures de câbles, ces dernières sont exposées à devenir conductrices de courants de plusieurs dizaines de milliers de volts. Il y a alors péril pour le poisson et, ce qui est pis, pour le pêcheur.

(1) ...Under some circonstances success has been had with the electric screen ; but public opinion frequently is opposed to its use, and no larger program of future installation of this type is contemplated... - Annual report of the Commissionner of Fisheries to the Secretary of Commerce for the fiscal year endef June 301932 , p. XXIII. - Government jrinting Office, Washington, 1932. 